\title{
Granzyme B leakage-induced apoptosis is a crucial mechanism of cell death in nasal-type NK/T-cell lymphoma
}

\author{
Young-Hyeh Ko ${ }^{1,2}$, Sanghui Park' ${ }^{1}$, Heekyung Jin², Heesook Woo ${ }^{1}$, Hyunseop Lee ${ }^{1}$, Chaewha Park ${ }^{3}$ and Kihyun Kim ${ }^{3}$
}

This study aims to investigate the role of granzyme B in the apoptosis of nasal-type NK/T-cell lymphoma. Twenty-four nasal-type NK/T-cell lymphomas were examined by TdT-mediated deoxyuridine triphosphate (dUTP)-biotin nick-end labeling (TUNEL) assay and immunohistochemical staining for active caspase 3, poly(ADP-ribose) polymerase (PARP-1/ p85)/p85, and Bcl-2. In addition, HANK-1 and NKL cell lines were analyzed using Western blot analysis. Immunoprecipitation was performed to identify the binding of granzyme B and intrinsic serpin proteinase inhibitor 9 (PI-9). To localize granzyme $B$, immunogold labeling and immunofluorescence staining were performed. The expression level of granzyme $B$ in tumor tissue was correlated with the apoptosis rate $(P=0.015)$, degree of necrosis $(P=0.002)$, and the levels of active caspase $3(P=0.036)$ and poly ADP-ribose polymerase (PARP)-1/p85 $(P=0.040)$. The granzyme B-positive HANK-1 cell line showed increased spontaneous cell death compared to the granzyme B-negative NKL cell line. The untreated HANK-1 cells released cytochrome $c$ into the cytosol with cleavage of caspase 3 and PARP-1. Treatment with granzyme B inhibitor and caspase inhibitor decreased the cleavage of PARP-1. By performing immunogold labeling, granzyme B was identified within the cytolytic granules as well as in the cytosol. Confocal microscopy and immunoprecipitation assays confirmed the colocalization of PI-9 and granzyme B, which formed an SDS-resistant complex. These results suggested that granzyme B leakage induces cell death in NK/T-cell lymphomas via both caspase-dependent and -independent mechanisms, and this leads to the extensive necrosis that is commonly seen in NK/T-cell lymphoma. Laboratory Investigation (2007) 87, 241-250. doi:10.1038/labinvest.3700517; published online 29 January 2007

KEYWORDS: apoptosis; granzyme B; NK/T-cell lymphoma

Nasal-type NK/T-cell lymphoma is a neoplasm of cytotoxic NK cells together with a minority of cytotoxic $T$ cells, and virtually all the cases are latently infected by Epstein-Barr virus (EBV). Histologically, the tumor is characterized by extensive coagulative necrosis and common apoptotic bodies. ${ }^{1}$

Angiocentricity with angioinvasion of the NK cell neoplasm has been proposed as a cause of necrosis, ${ }^{2}$ but the absence of histological evidence for angioinvasion in many of these cases has also implicated other factors such as the Fas/ Fas ligand system and chemokines such as monokine induced by interferon- $\gamma$ (Mig) and interferon- $\gamma$ inducible protein-10 (IP-10). ${ }^{3}$ The Fas/Fas ligand system is an important mechanism for triggering apoptosis. As Fas and Fas ligand are expressed in most cases of NK/T-cell lymphoma, ${ }^{4}$ it is con- ceivable that the Fas/Fas ligand system contributes to the commonly seen apoptosis/necrosis of NK/T-cell lymphoma. However, NK/T-cell lymphoma is resistant to Fas-mediated apoptosis because of the common Fas gene mutation and the expression of antiapoptotic proteins in NK/T-cell lymphoma. ${ }^{5,6}$ Accordingly, the Fas/Fas ligand expression in NK/Tcell lymphoma tissue has no correlation with necrosis. ${ }^{4}$

IP-10 and Mig are predominantly expressed in the endothelial cells, lymphocytes, and macrophages that surround the necrosis. ${ }^{3}$ In vitro, IP-10 enhances NK-cell-mediated cell lysis and the release of granule-derived serine esterases. ${ }^{7}$

Granzyme B is one of the lymphocyte serine proteases and targets caspase 3 directly or indirectly through the mitochondria; this initiates a caspase cascade that results in DNA fragmentation and apoptosis. In cytolytic T and NK

\footnotetext{
${ }^{1}$ Department of Pathology, Samsung Medical Center, Sungkyunkwan University School of Medicine, Seoul, Korea; ${ }^{2}$ Samsung Biochemical Research Institute, Seoul, Korea and ${ }^{3}$ Division of Hematology-Oncology, Internal Medicine, Samsung Medical Center, Sungkyunkwan University School of Medicine, Seoul, Korea Correspondence: Dr Y-H Ko, MD, Department of Pathology, Samsung Medical Center, Sungkyunkwan University, 50 Ilwon-dong, Kangnam-gu, Seoul 135-710, Korea. E-mail: yhko@smc.samsung.co.kr

This study was presented at the 95th Annual Meeting of the United States and Canadian Academy of Pathology, Atlanta, Georgia, February 11-17, 2006.

Received 11 June 2006; revised 28 October 2006; accepted 25 November 2006
} 
cells, granzyme B is localized within the cytoplasmic granules; however, once it is released from the cytolytic T cells, granzyme B binds to its target cell receptors and is then endocytosed. ${ }^{8}$ Granzyme B is expressed in virtually all cases of NK/T-cell lymphoma. ${ }^{9}$ Although cytolytic T or NK cells are protected from the toxic effect of intrinsic granzyme $\mathrm{B}$, because granzyme B is generally confined within cytolytic granules and the cell is protected by intrinsic serpin proteinase inhibitor 9 (PI-9), ${ }^{8,10}$ it is possible that granzyme B attacks the cell from within, and this leads to self-induced death of the NK/T lymphoma cells. In an attempt to verify this hypothesis, we analyzed the relationship between granzyme B and the observed cell death in histologic sections of NK/T-cell lymphoma. We also investigated the effect of granzyme B on the cell death in an NK/T-cell lymphoma cell line. Finally, an immunoelectron microscopic study and immunofluorescence staining with immunoprecipitation analysis were performed to explore the pathogenetic mechanism by which granzyme B causes self-induced death of NK tumor cells.

\section{MATERIALS AND METHODS Analysis of Cell Death and the Related Protein in Tumor Tissue with Clinical Correlation \\ Patient material}

Formalin-fixed, paraffin-embedded tissues of 24 nasal-type NK/T-cell lymphomas were retrieved from the surgical pathology files. The diagnosis of nasal-type NK/T-cell lymphoma was based on the recent World Health Organization classification. ${ }^{1}$ By definition, all cases were positive for EBV by in situ hybridization.

\section{Detection of apoptosis}

Apoptosis was analyzed by performing a TdT-mediated deoxyuridine triphosphate (dUTP)-biotin nick-end labeling (TUNEL) assay using the ApopTag-Peroxidase in situ apoptosis detection kit (CHEMICON International, Inc., Temecula, CA, USA). Positive staining was observed in the nuclei of the apoptotic cells and in the apoptotic bodies. The percentages of the TUNEL-positive cells were quantified by using a video overlay-based system. The apoptosis rate (AR) was expressed as the percentages of positive tumor nuclei and apoptotic bodies among all the nuclei present in a tissue section, as judged by the morphology. Closely located apoptotic bodies that appeared to be derived from a single nucleus were counted as one. At least 10 or more high-power fields $(\times 400)$ were analyzed for each case.

\section{Immunohistochemical detection of the apoptosis-related protein}

The paraffin-embedded tissues were cut into $4 \mu \mathrm{m}$ sections and then stained by an automated stainer (Techmate 1000, DakoCytomation) using a standard peroxidase-antiperoxidase method with 3,3'-diaminobenzidine as a chromogen. The following antibodies were used: monoclonal antibody for granzyme B (Zymed, South San Francisco, CA, USA; 1:40), Bcl-2 (Dako, Glostrup, Denmark; 1:40), and polyclonal rabbit antibody for active caspase 3 (BD Pharmingen, San Diego, CA, USA; 1:50) and human poly(ADPribose) polymerase (PARP-1/p85) (Promega, Madison, WI, USA; $1: 100$ ). For caspase 3 and PARP-1/p85, only those cells with nuclear staining were counted. The percentages of active caspase-3-positive cells and PARP-1/p85-positive cells were quantified using a video overlay-based system. Semiquantitative analysis was performed to evaluate the stains for granzyme B and Bcl-2. Stains for granzyme B were divided into four grades: grade 1, positive staining in only occasional cells or a few cells; grade 2, weak staining in more than 50\% of tumor cells; grade 3, strong staining with a well-defined granular pattern in more than $50 \%$ of tumor cells; grade 4 , strong staining with a dispersed granular pattern in more than $50 \%$ of tumor cells. The tumor cells showed cytoplasmic staining for Bcl-2. The cases showing positive reaction in more than $10 \%$ of tumor cells were regarded as being positive.

\section{Statistical analysis}

Correlations were calculated using a Pearson correlation test or a Spearman correlation test. All values are based on twotailed statistical analysis and $P$-values less than 0.05 were considered significant. All statistical analyses were performed using the SPSS software (version 10.0, SPSS, Chicago, IL, USA).

\section{Analysis of Cell Death in the NK/T-Cell Lymphoma Cell Line \\ Cell lines}

Three cell lines were used. The HANK-1 cell line (kindly provided by Dr Kagami and Dr Seto, Aichi Cancer Center, Nagoya, Japan) was established from a nasal-type NK/T-cell lymphoma that was latently infected with EBV and it was cultured in COS medium (Cosmo Bio, Tokyo, Japan) supplemented with $5 \%$ human plasma and $100 \mathrm{U} / \mathrm{ml}$ human recombinant IL-2 (Biosource International, Camarillo, CA, USA) in a $5 \% \mathrm{CO}_{2}$ atmosphere at $37^{\circ} \mathrm{C}^{11}$ The NKL cells (kindly provided by Dr Seto, Aichi Cancer Center, Japan) were established from a $\mathrm{CD} 16+\mathrm{CD} 56+$ large granular lymphocyte leukemia and maintained in RPMI 1640 supplemented with $10 \%$ fetal bovine serum, $100 \mathrm{U} / \mathrm{ml}$ penicillin G, $100 \mu \mathrm{g} / \mathrm{ml}$ streptomycin, and $100 \mathrm{U} / \mathrm{ml}$ recombinant IL-2. ${ }^{12}$ Jurkat $\mathrm{T}$ cells were maintained in RPMI 1640 supplemented with $10 \%$ fetal bovine serum, $100 \mathrm{U} / \mathrm{ml}$ penicillin $\mathrm{G}$, and $100 \mu \mathrm{g} / \mathrm{ml}$ streptomycin.

\section{Analysis of apoptosis}

Apoptosis was assessed by flow cytometry using FITC-conjugated Annexin $\mathrm{V}$ and propidium iodide double-staining (R\&D Systems, Minneapolis, MN, USA). The early-stage apoptosis was FITC-positive but PI negative, and late apoptotic/necrotic cells were both FITC positive and PI positive. 
Treatment with etoposide

To induce apoptosis, cells $\left(1 \times 10^{6}\right.$ cells/well $)$ were seeded onto a six-well plate and then treated with etoposide at $10 \mu \mathrm{M}$ final concentration (Sigma, St Louis, MO, USA). After incubation for $0,1,2,3$, and $6 \mathrm{~h}$, the cells were harvested and analyzed.

\section{Treatment with inhibitors for granzyme $B$ and caspase}

The HANK-1 cells were treated with $40 \mu \mathrm{M}$ Z-AAD-CMK (Calbiochem, La Jolla, CA, USA) and Z-VAD-FMK (Sigma, St Louis, MO, USA). After incubation for $72 \mathrm{~h}$, the degree of apoptosis and activation of caspase 3 and PARP-1 were compared with the untreated HANK-1 cells.

\section{Measurement of mitochondrial membrane potential}

The measurement of mitochondrial membrane potential $\left(\Delta \Psi_{\mathrm{m}}\right)$ was performed with a JC-1 Kit (Biotium Inc., Hayward, CA, USA) using the manufacturer's guidelines. Cells $\left(1 \times 10^{6}\right)$ were incubated with $5 \mu \mathrm{g} / \mathrm{ml} \mathrm{JC}-1$ at $37^{\circ} \mathrm{C}$ for $20 \mathrm{~min}$ and analyzed by flow cytometry (PAS, Partec, Münster, Germany).

\section{Cytochrome c release assay}

The mitochondrial and cytosolic fractions of the cells were obtained as previously described, ${ }^{13}$ and immunoblotting for cytochrome $c$ was performed. The membrane was stripped and reprobed with anti-COX IV $\mathrm{mAb}$ as a mitochondrial marker and to demonstrate equal protein loading.

Western blotting to detect active caspase 3, cleaved PARP-1, cytochrome $\mathrm{C}$, and granzyme $B$

Cellular proteins were extracted from the cell lines using Pro-Prep $^{\mathrm{TM}}$ (Intron, Sungnam-Si, Korea). Immunoblotting was performed using polyclonal antibodies for active caspase 3 and cleaved PARP-1/p85, using monoclonal antibodies for cytochrome $c$ (BD Pharmingen ${ }^{\mathrm{TM}}$, San Diego, CA, USA), granzyme B (Zymed, South San Francisco, CA, USA), and COX-IV (Abcam, Cambridge, UK), and detected using the ECL system (PIERCE, Rockford, IL, USA).

\section{Immunoprecipitation assay to detect granzyme B/PI-9 complex}

HANK-1 cells and NKL cells were extracted with 1\% NP40 lysis buffer (1\% NP40, $50 \mathrm{mM}$ Tri-Cl pH 8.0, $150 \mathrm{mM} \mathrm{NaCl}$, $0.1 \%$ SDS, and $0.02 \%$ sodium azide) containing $2 \mathrm{mM} \mathrm{Zn}^{2+}$. Immunoprecipitation was performed in a $4{ }^{\circ} \mathrm{C}$ cold room for $16 \mathrm{~h}$ with constant stirring. The precipitates were harvested by centrifuging for $15 \mathrm{~s}$ at 14000 r.p.m. in a refrigerated Eppendorf microcentrifuge. After electrophoresis on SDSpolyacrylamide gels, the protein was detected with mouse anti-granzyme B antibody (Zymed, South San Francisco, CA, USA), and it was visualized using the ECL system (PIERCE, Rockford, IL, USA). To confirm the result, the reverse procedure was carried out using immunoprecipitation of granzyme B followed by immunoblotting with anti-PI-9 antibody.
Confocal microscopy to detect the colocalized granzyme B/PI-9

Cytospin slides made from the HANK-1 cell line were incubated with the primary antibody against PI-9 (1:100, Santa Cruz Biotechnology, Inc., Santa Cruz, CA, USA) for $1 \mathrm{~h}$. The slides were then incubated with goat anti-mouse secondary antibody conjugated with Alexa Fluor (Molecular Probes, Invitrogen Detection Technologies, Carlsbad, CA, USA) for $1 \mathrm{~h}$. The slides were then washed with phosphate-buffered solution and incubated with the primary antibody against granzyme B (Zymed, South San Francisco, CA, USA; 1:40) for $1.5 \mathrm{~h}$. After washing, secondary antibody conjugated with FITC was applied and this was allowed to incubate for a further $1 \mathrm{~h}$. The slides were analyzed using a confocal laserscanning microscope (Leica Microsystems, Bannockburn, IL, USA).

\section{Electron microscopic study with immunogold labeling to detect granzyme $B$ leakage}

Ultrathin sections $(5-60 \mathrm{~nm})$ of HANK-1 cells were incubated with a monoclonal granzyme B antibody (Zymed, CA, USA; 1:20) in serum-free protein block (DakoCytomation, Glostrup, Denmark) for $1.5 \mathrm{~h}$. The section was then incubated for $1.5 \mathrm{~h}$ at $36^{\circ} \mathrm{C}$ with goat antimouse IgG conjugated to $10 \mathrm{~nm}$ gold particles diluted in serum-free protein block (British-Biocell, London, UK; 1:10). After washing in serum-free protein block followed by PBS and distilled water, the sections were counterstained with uranyl acetate and lead citrate. The control sections were incubated with mouse serum instead of the primary antibody, and showed negative results.

\section{RESULTS \\ Analysis of Cell Death and the Related Protein in Tumor Tissue}

The apoptosis rate is correlated with the degree of necrosis, the size of the tumor cells, and the level of active caspase 3, and it is inversely correlated with $\mathrm{Bcl}-2$ (Table 1)

The percentage of TUNEL assay-positive cells ranged from 0.6 to $56.4 \%$ with a mean of $7.4 \%$. The optimal cutoff values for the percentage of TUNEL-positive cells were determined using the log-rank test. The level of apoptosis and presence of necrosis were significantly correlated $(P=0.039)$. The percentage of active caspase 3-positive cells ranged from 0 to $17 \%$ with a mean of $2.8 \%$. Using a $2 \%$ cutoff value, correlation was noted between the apoptosis rate and expression of active caspase $3(P=0.034)$. Tumors with a high apoptosis rate showed a greater number of active caspase 3-positive cells. The level of PARP-1/p85 was strongly correlated with the level of the active caspase 3 expression (Spearman's correlation, $P=0.008$ ), which suggests that apoptosis in NK/ T-cell lymphomas involves activation of effector caspase 3 and cleavage of one of the major effector caspase substrates, PARP-1. Bcl-2 was detected in the cytoplasmic staining of the tumor cells. The percentage of Bcl-2-positive tumor cells 
ranged from 2 to $70 \%$. Only nine out of 24 cases expressed Bcl-2 in more than $10 \%$ of the tumor cells. Using a $10 \%$ cutoff value, an inverse correlation was identified between Bcl-2 expression $(>10 \%)$ and the apoptosis rate $(P=0.009)$.

Table 1 Correlation of apoptosis analyzed by TUNEL assay with pathological parameters in $24 \mathrm{NK} / \mathrm{T}$-cell lymphoma tissues

\begin{tabular}{lc}
\hline Parameters & Apoptosis rate \\
\cline { 2 - 3 } & $<$-value \\
\hline
\end{tabular}

\section{Necrosis}

Absent

3

9

Active caspase 3 (\%)

$\begin{array}{rrr}<2 & 11 & 6 \\ >2 & 1 & 6\end{array}$

PARP-1 index

Median

$0.88 \%$

$4.6 \%$

$>0.05$

$\mathrm{BCl}-2$ (\%)

$<10$

$>10$
4

8
11
The expression level of granzyme $B$ is significantly correlated with apoptosis rate, active caspase 3 , and PARP-1/p85

(Figure 1a-c)

Granzyme B stained at variable intensities and in various proportions of the tumor cells. The staining pattern was granular; some cases showed well-delineated coarse granules localized to the cytoplasmic area, but other cases showed dispersed fine or coarse granules of variable size throughout the entire cytoplasm. Because granzyme B targets caspase 3 directly or indirectly through the mitochondria, the relation between granzyme B and active caspase 3 was analyzed. When considering the cases showing strong staining in more than $50 \%$ of the tumor cells (grades 3 and 4), a strong expression of granzyme $\mathrm{B}$ was correlated with the apoptosis rate $(P=0.015)$, the expression of active caspase $3(P=0.036)$ and PARP-1/p85 $(P=0.040)$, but it was not correlated with $\mathrm{Bcl}-2(P=0.092)$. These results suggest that granzyme $\mathrm{B}$ plays a role in the apoptosis of NK/T-cell lymphoma.

The level of granzyme B is significantly correlated with the degree of necrosis of the tumor tissue (Figure 1d)

To evaluate the role of granzyme B in the necrosis of NK/Tcell lymphoma, the degree of necrosis was correlated with the grade of the granzyme B staining. Granzyme B staining was evaluated in the tumor tissue that was distal from the necrosis as well as in the tumor tissue that was proximal to the necrosis. The degree of tumor necrosis was divided into four grades according to the severity. Fourteen of 24 cases showed a variable degree of necrosis. The degree of necrosis was significantly correlated with the level of granzyme B
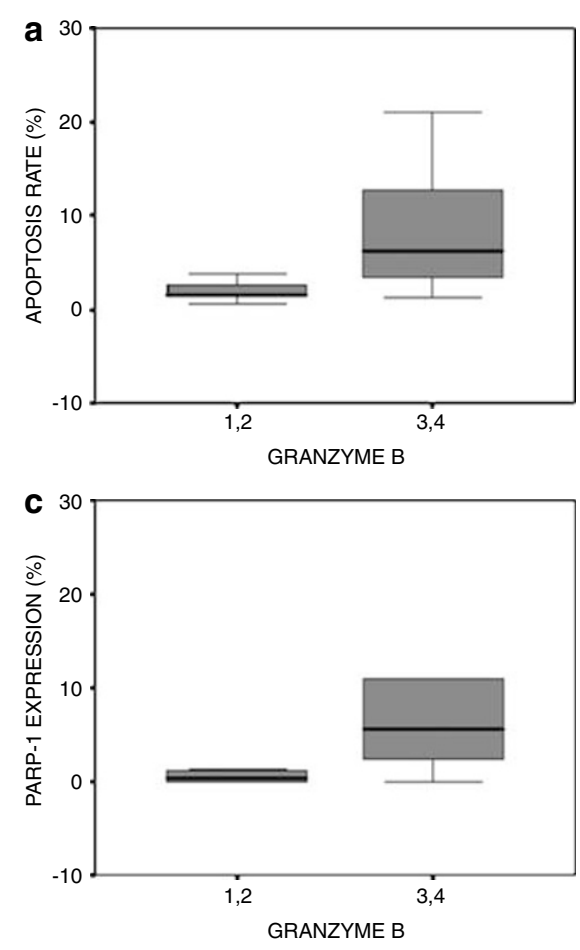
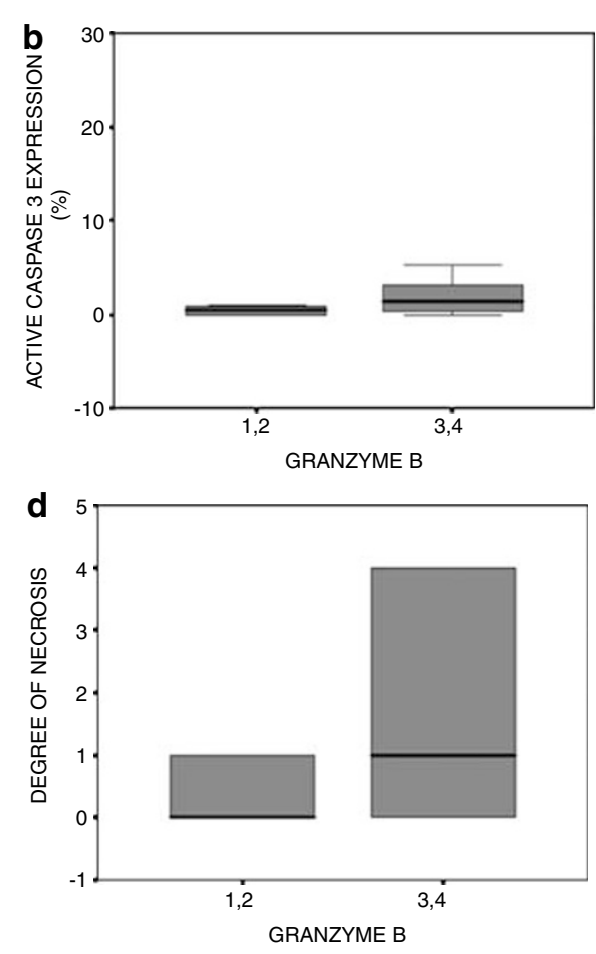

Figure 1 Correlation of the level of granzyme B with apoptosis, active caspase 3 , PARP-1/p85, and necrosis. The cases with strong immunohistochemical reactivity for granzyme B, in more than $50 \%$ of the tumor cells (grades 3 and 4 ), showed higher apoptosis rate $(P=0.015)(\mathbf{a})$, increased expression of active caspase $3(P=0.036)(\mathbf{b})$, and PARP-1/p85 $(P=0.040)(\mathbf{c})$. The degree of tumor necrosis was divided into four grades according to the severity. The cases with higher granzyme $B$ level showed more extensive degree of necrosis $(P=0.002)(\mathbf{d})$. 
expression in the tumor tissue surrounding the necrosis $(P=0.002)$ as well as in the more remote tumor tissue $(P=0.019)$. Granzyme B staining tended to be stronger and more dispersed throughout the cytoplasm in the proximal peri-necrotic tumor tissue than in the distal tumor tissue.

\section{Analysis of Cell Death in the NK/T-Cell Line}

To verify the relation of granzyme $\mathrm{B}$ and increased cell death in NK/T-cell lymphoma, we examined the HANK-1 cell line, which contains abundant granzyme B. As a control, the NKL cell line or Jurkat cell line, which is devoid of granzyme B in the unstimulated state, was used.

The granzyme B-positive HANK-1 cell line shows a significant degree of spontaneous cell death with mitochondrial alteration and activation of caspase 3 (Figures 2-5)

We first compared the rates of cell death between the HANK1 and NKL cell lines by performing annexin V/PI doublestaining-based flow cytometric analysis. Both cell lines were cultured in the same conditions with IL-2. As shown in Figure 2, the HANK-1 cell line showed that significantly larger numbers of cells underwent spontaneous apoptosis than did the NKL cell line. As granzyme B induces apoptosis via the mitochondrial pathway with direct activation of caspase 3, we examined the activation of caspase 3, the cleavage of PARP-1, loss of $\Delta \Psi_{\mathrm{m}}$, and the release of cytochrome $c$. As shown in Figure 3, the HANK-1 cell line exhibited cleavage of caspase 3 into 17 and $19 \mathrm{kDa}$ forms before, as well as after, the administration of $10 \mu \mathrm{M}$ etoposide. Contrary to the HANK-1 cell line, the NKL cells showed the $17 \mathrm{kDa}$ cleaved form of caspase 3 only $2 \mathrm{~h}$ after treatment with etoposide. A similar finding was observed for the PARP$1 / \mathrm{p} 85$ protein (Figure 3). Loss of $\Delta \Psi_{\mathrm{m}}$ was observed in many untreated HANK-1 cells; this was seen for $53.05 \%$ of the cells we analyzed, whereas only $8.05 \%$ of the untreated NKL cells lost their $\Delta \Psi_{\mathrm{m}}$, which is in accord with the increased apoptosis in the HANK-1 cell line (Figure 4). Using Western blotting, cytochrome $c$ was observed in the cytosolic fraction as well as in the mitochondrial fraction of the untreated HANK-1 cell line, indicating that spontaneous apoptosis of the HANK- 1 cells was mediated by the cytochrome $c$ released from the mitochondria into the cytosol (Figure 5).

Granzyme B inhibitor suppresses the cleavage of PARP-1/p85 with a partial block of the mitochondrial $\Delta \Psi_{m}$

(Figures 6 and 7)

To verify the effect of granzyme B on the cell death of the HANK-1 cells, we administered granzyme B inhibitor (Z-AAD-CMK) to the HANK-1 cells and then incubated them for 3 days. The Z-AAD-CMK-treated HANK-1 cells showed decreased cleavage of PARP-1 and partial blockage of the $\Delta \Psi_{\mathrm{m}}$ loss. Caspase inhibitor decreased the cleavage of PARP-1, but had no effect on the $\Delta \Psi_{\mathrm{m}}$ loss and the
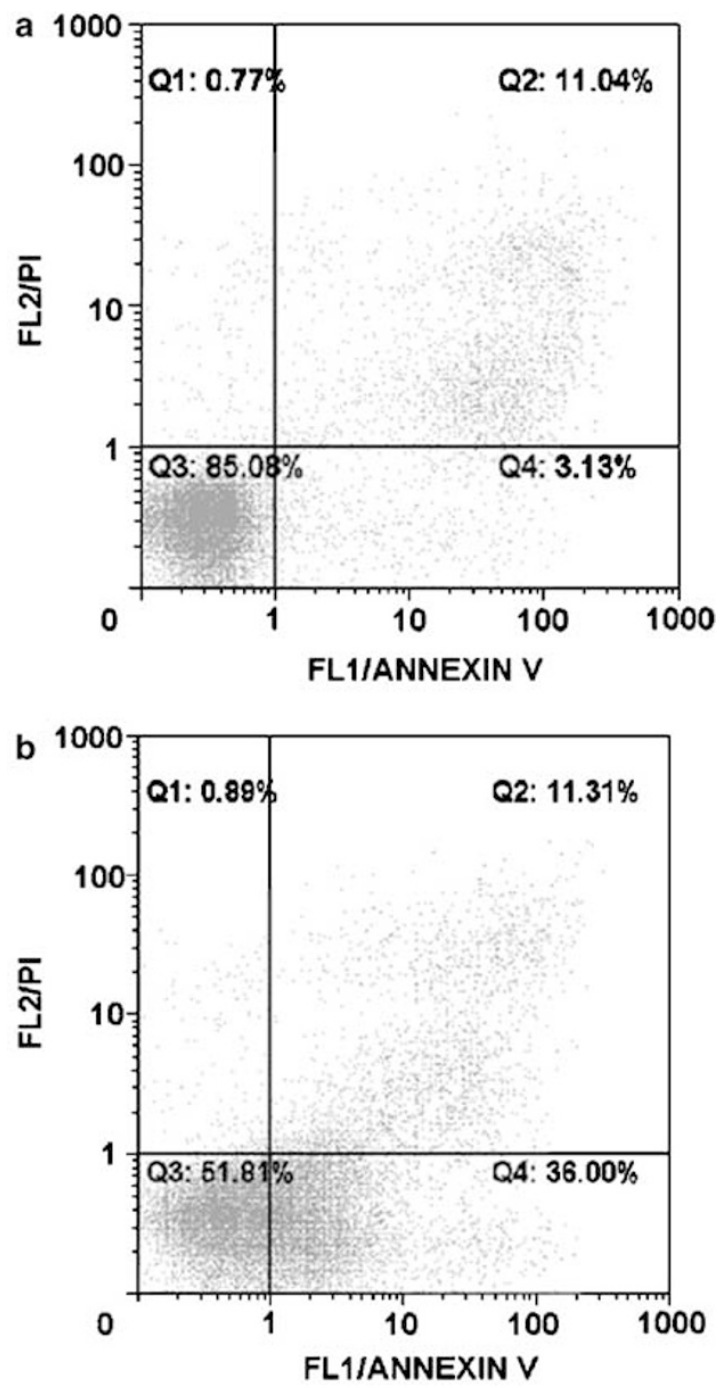

Figure 2 Flow cytometric analysis of apoptotic cells using ANNEXIN V/PI double stain in untreated NKL cell (a) and HANK-1 cell (b) lines. ANNEXIN Vpositive- and PI-negative cells are in an early stage of apoptosis and are still viable. The cells stained with both PI and ANNEXIN V are either in the later stage of apoptosis or are already dead. The HANK-1 cell line showed that significantly larger numbers of cells underwent spontaneous apoptosis than did the NKL cell line (36 vs 3.13\%).

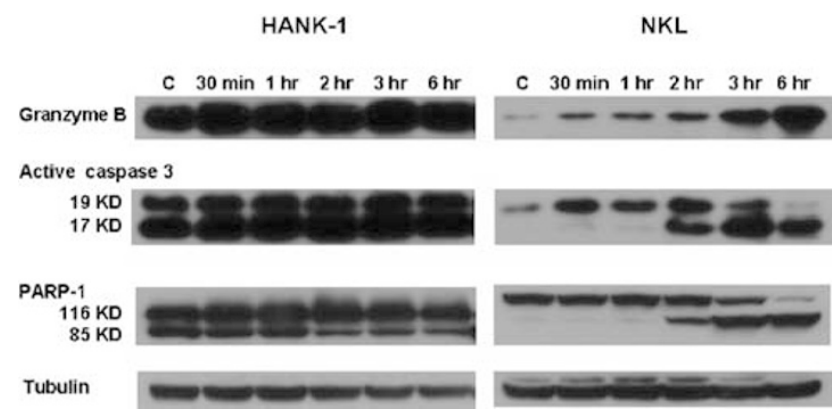

Figure 3 HANK-1 cell line exhibits cleavage of caspase 3 into 17 and $19 \mathrm{kDa}$ fragments before, as well as after, the administration of $10 \mu \mathrm{M}$ etoposide. Contrary to findings for HANK-1, NKL cells showed the $17 \mathrm{kDa}$ cleaved form of caspase 3 only $2 \mathrm{~h}$ after etoposide treatment. A similar finding was observed for PARP-1/p85 protein. 

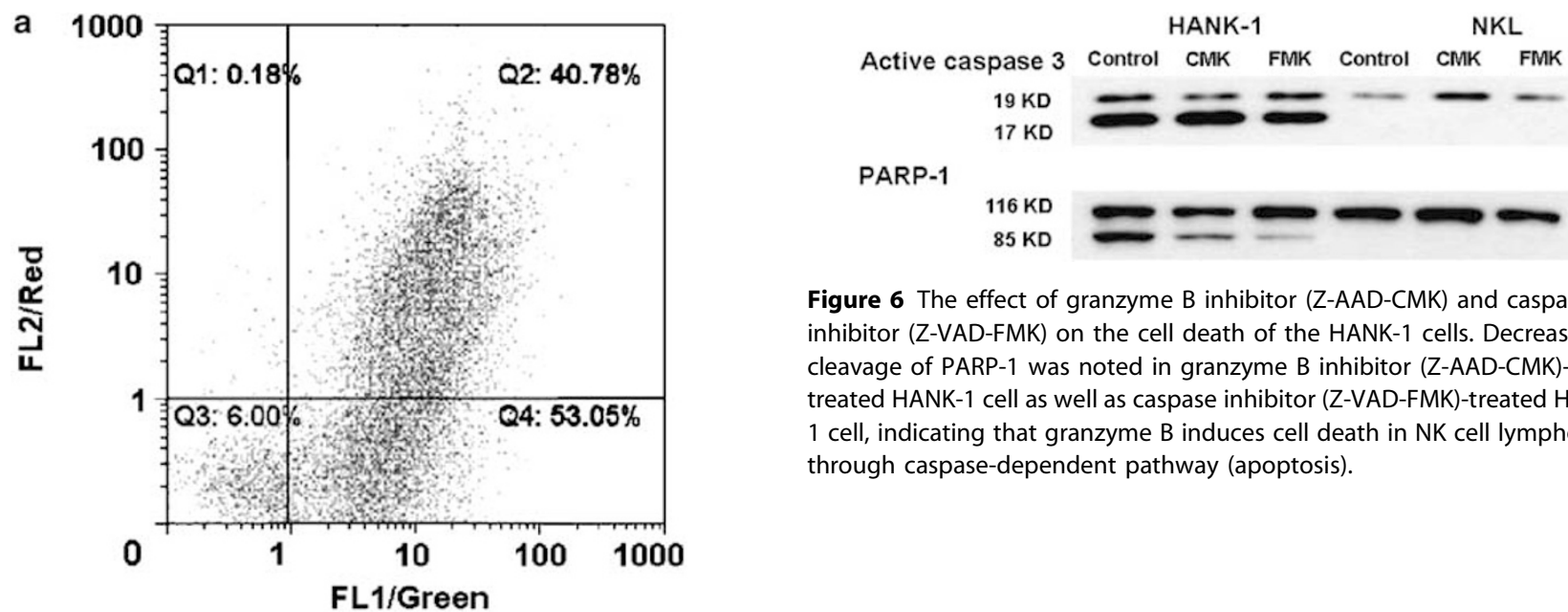

Figure 6 The effect of granzyme B inhibitor (Z-AAD-CMK) and caspase inhibitor (Z-VAD-FMK) on the cell death of the HANK-1 cells. Decreased cleavage of PARP-1 was noted in granzyme $B$ inhibitor (Z-AAD-CMK)treated HANK-1 cell as well as caspase inhibitor (Z-VAD-FMK)-treated HANK1 cell, indicating that granzyme B induces cell death in NK cell lymphomas through caspase-dependent pathway (apoptosis).

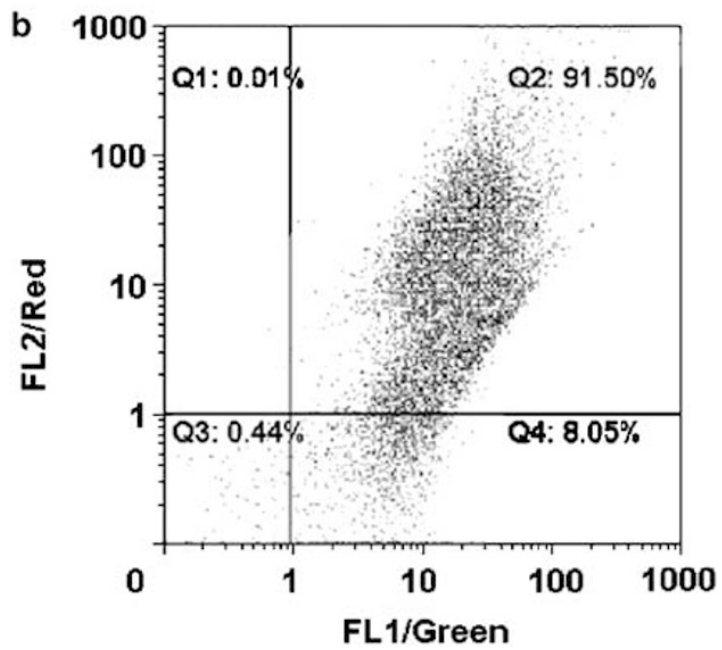

Figure 4 Mitochondrial staining using JC-1 with flow cytometric analysis for measurement of $\Delta \Psi_{\mathrm{m}}$. A dot plot of red fluorescence (FL2) vs green fluorescence (FL1) resolved live cells from apoptotic and dead cells. Mitochondria containing red JC- 1 aggregates in healthy cells are detectable in the FL2 channel, and green JC-1 monomers in apoptotic cells are detectable in the FITC channel (FL1). Note the increase of dead cell numbers with decreased red fluorescence in HANK-1 cell line (a) compared to NKL cell line (b) (53.05 vs $8.05 \%)$.

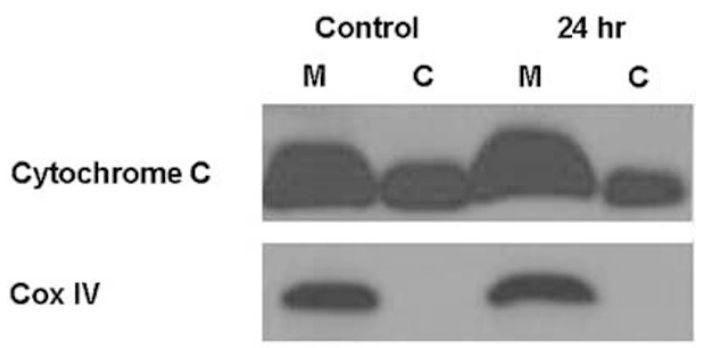

Figure 5 Cytochrome $c$ release assay. Cytochrome $c$ was observed by Western blotting in the cytosolic fraction as well as in the mitochondrial fraction of the HANK-1 cell line, which indicates that spontaneous apoptosis of HANK-1 cells was mediated by cytochrome $c$ (Control: untreated HANK-1 cells, $24 \mathrm{~h}$; after treatment with etoposide).
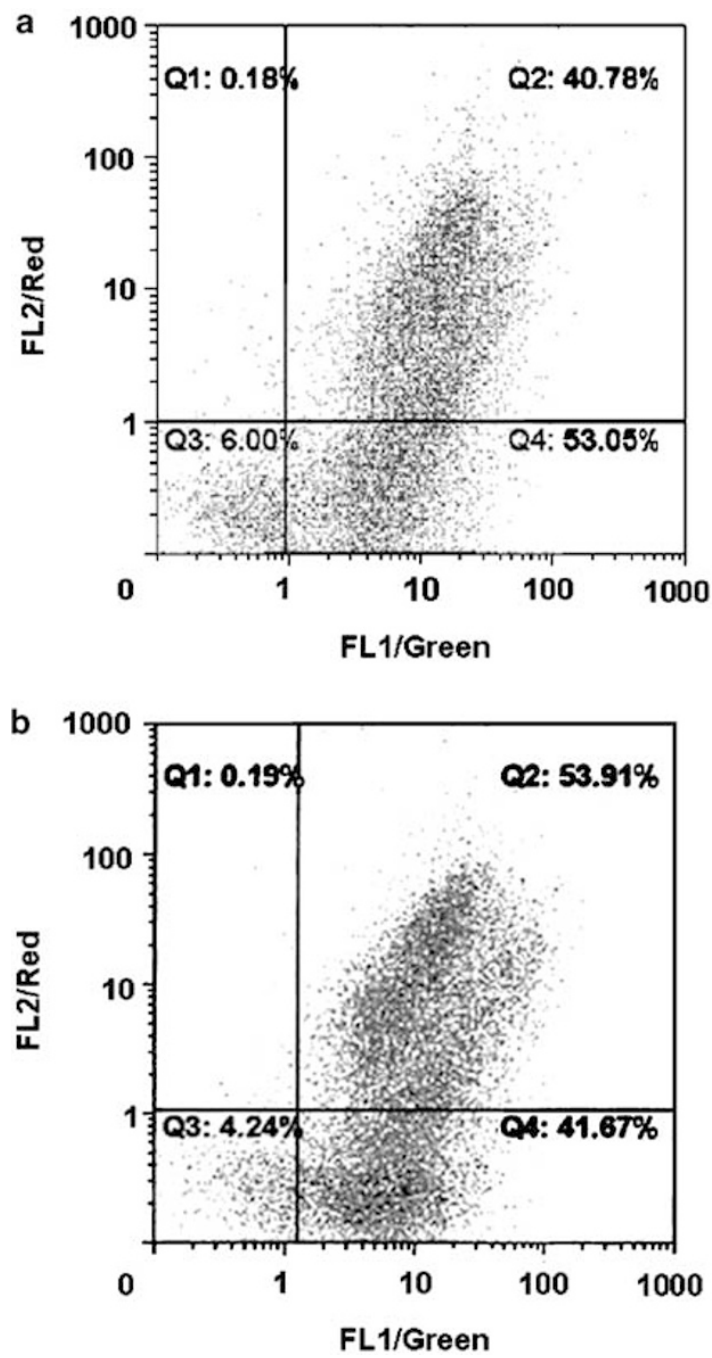

Figure 7 Mitochondrial staining using JC-1 with flow cytometric analysis to evaluate the effect of granzyme $B$ inhibitor on loss of $\Delta \Psi_{\mathrm{m}}$. A dot plot of red fluorescence $(F L 2)$ vs green fluorescence (FL1) resolved live cells from apoptotic and dead cells with decreased $\Delta \Psi_{\mathrm{m}}$. Compared to control HANK1 cell line (a; 53.05\%), HANK-1 cells treated with granzyme B inhibitor showed the decrease of apoptotic and dead cell numbers (b; $41.67 \%)$, indicating that at least a part of granzyme B induced-cell death in NK/T-cell lymphoma is through mitochondrial pathway (necrosis). 
release of cytochrome $c$. These findings suggest that granzyme B induces cell death in NK cell lymphomas through both caspase-dependent and caspase-independent mechanisms.

Granzyme B is located in the cytosol as well as in the cytolytic granules of HANK-1 cells (Figure 8)

In cytolytic cells, granzyme B is stored in the cytoplasmic granules, enters into the target cells via receptor-binding endocytosis, and is released into the cytosol by perforin mediation. Cytolytic cells are protected from the endogenous activity of granzyme B because of the enzyme's localization within the membrane-bound granules and the endogenous inhibitor, PI-9. However, activated cytolytic cells can leak granzyme B into the cytosol, which can lead to self-lysis of cytolytic cells. We examined the ultrastructural distribution of granzyme B in HANK-1 cells to identify the leakage of granzyme B. Using immunogold labeling, granzyme B labeled with gold particles was commonly found in the membranebound dense cytolytic granules; however, a significant number of gold particles in the free space of the cytoplasm was observed.

\section{Detection of granzyme B/granzyme B inhibitor (PI-9) complex (Figure 9)}

The distribution of granzyme B inhibitor, PI-9, was distinct from that of granzyme B, and it was localized in the cytosol. When granzyme B was released into the cytosol, PI-9 neutralized the protease activity of granzyme B by forming an SDS-resistant intermolecular complex. We examined the formation of the granzyme B/PI- 9 complex by performing immunoprecipitation of the HANK-1 cell lysates using a monoclonal antibody reactive with granzyme B; this was followed by blotting with a PI-9-reactive antibody. As shown in Figure 9, granzyme B was detected as a $55-59 \mathrm{kDa}$ complex that was precipitated by PI-9; this confirmed that granzyme B was released from the cytolytic granules, allowing it to form an SDS-resistant complex with PI-9.

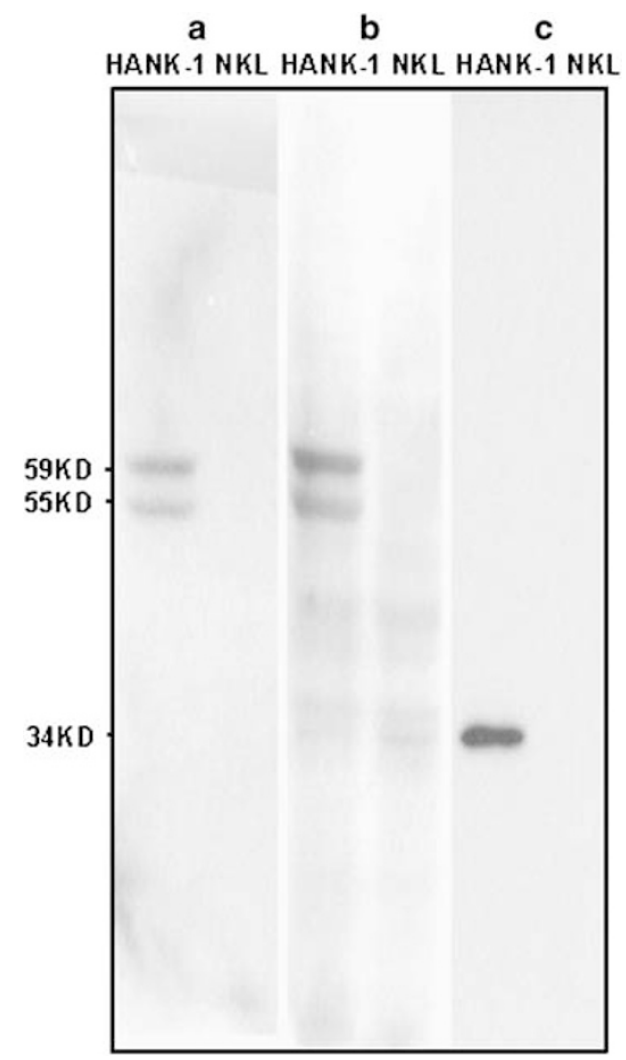

Figure 9 Detection of granzyme B/granzyme B inhibitor (PI-9) complex in the HANK-1 cell line and NKL cell line by immunoprecipitation. ((a) Protein extracted by NP40 lysis buffer and immunoprecipitated by granzyme B followed by blotting with a PI-9-reactive antibody. (b) Reverse experiment. Immunoprecipitation by PI-9 followed by blotting with a granzyme B. Granzyme B-PI-9 complex was detected as a 55-59 kDa complex. (c) Control. Protein extracted by Proprep Intron lysis buffer. Granzyme B is detected as $34 \mathrm{kDa}$ ).
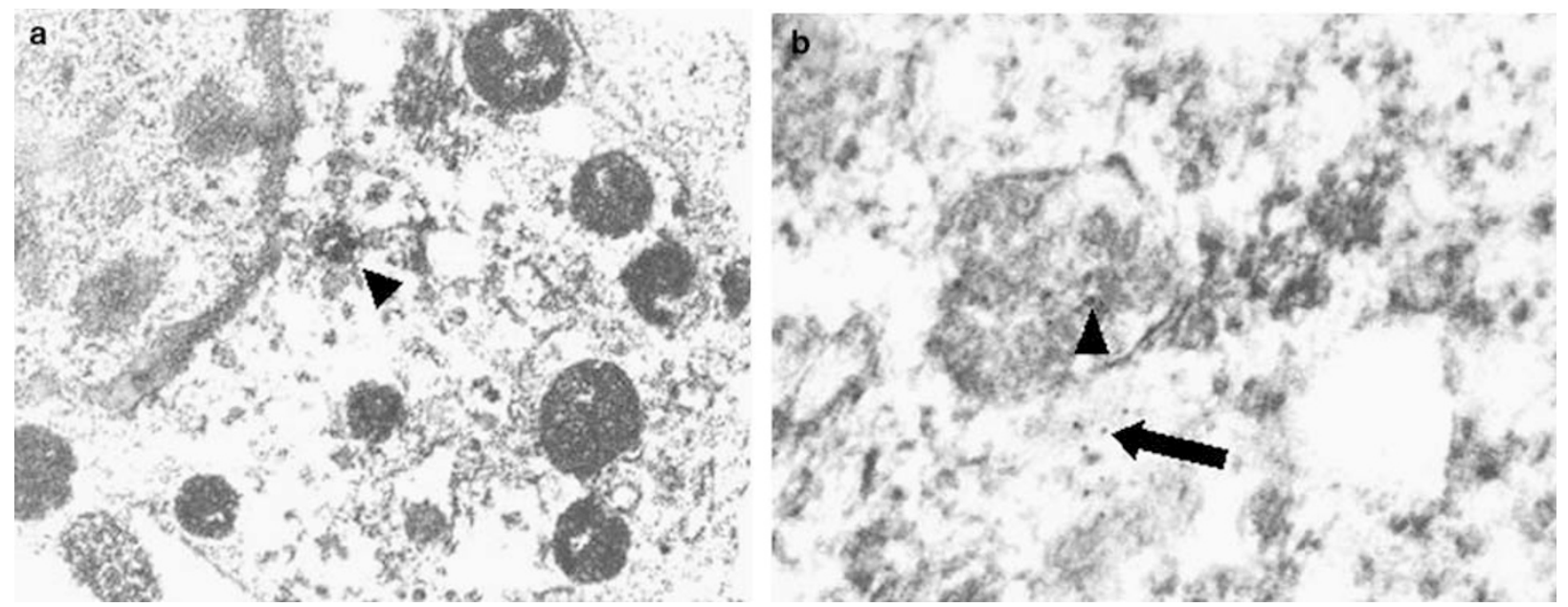

Figure 8 The ultrastructural distribution of granzyme B in HANK-1 cells demonstrated by immunogold labeling. Granzyme B labeled with gold particles was commonly found in the membrane-bound dense cytolytic granules (a and $\mathbf{b}$; arrow head); however, a significant number of gold particles in the free space of the cytoplasm was observed (b; arrow). 

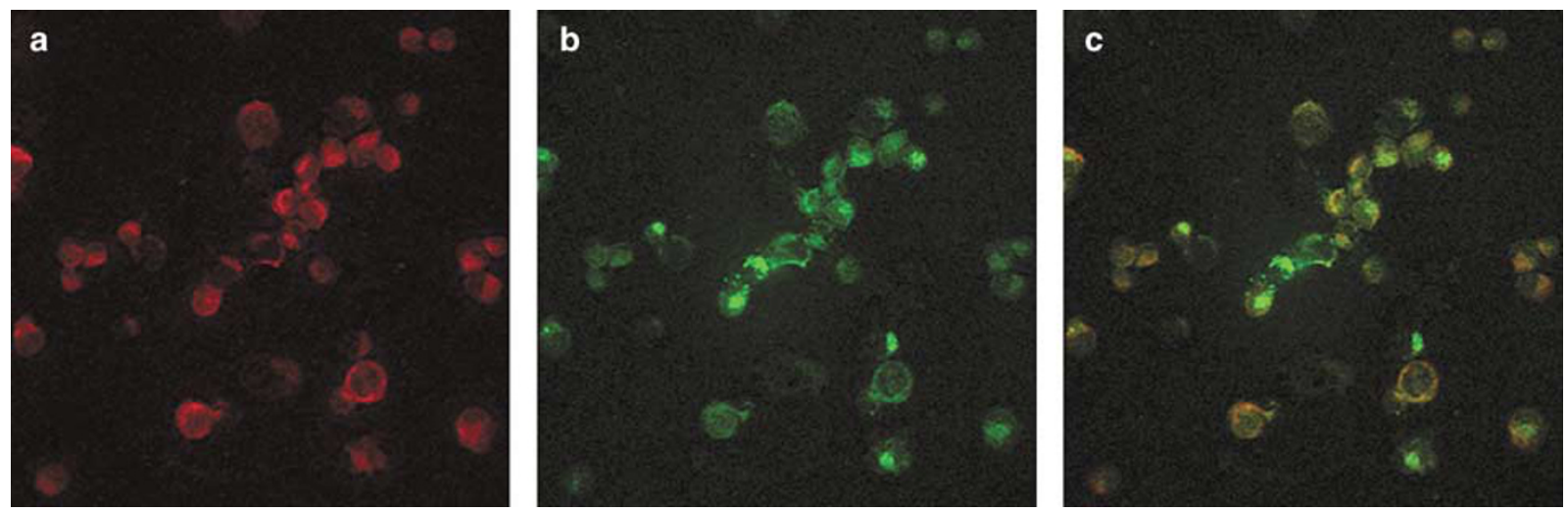

Figure 10 Immunofluorescence stain with PI-9 (a: red) and granzyme B (b: green) observed by confocal laser scanning microscopy. Note, PI-9 and granzyme B are colocalized (c: orange) in the cytoplasm of some cells.

Granzyme B and PI-9 are colocalized in the cytosol (Figure 10) To further demonstrate the leakage of granzyme $B$ into the cytosol to form a granzyme B/PI-9 complex, the HANK-1 cells were immunostained by double labeling with antibodies against granzyme B and PI-9; the cells were examined using confocal laser scanning microscopy. PI-9 and granzyme B displayed diffuse granular staining throughout the cytoplasm. Green-labeled granzyme B and red-labeled PI-9 were observed separately, as well as a fused orange signal, indicating colocalized PI-9 and granzyme B.

\section{DISCUSSION}

Apoptosis is characterized by certain distinctive morphologic and molecular changes such as cell shrinkage, chromatin condensation, and internucleosomal DNA fragmentation. ${ }^{14}$ Other typical features include phosphatidylserine exposure that is detected by an Annexin V assay. Its most classical form is exclusively observed when the caspases, particularly caspase 3 , are activated. ${ }^{15}$ On the other hand, necrosis is the common result of an irreversible cell injury from various causes. In contrast to apoptosis, where the morphologic features of injury are predominantly nuclear at the time of onset, necrotic cells exhibit concomitant nuclear and cytoplasmic disintegration. ${ }^{16}$

The zonal cell death commonly seen in the NK/T-cell lymphoma resembles coagulative necrosis, in which the dead tumor cells show only faint, ghost-like outlines with scattered nuclear dust, but careful observation commonly reveals that apoptotic cells are scattered among the completely necrotic tumor cells. This observation suggests that the zonal necrosis seen in NK/T-cell lymphomas may be formed through apoptosis as well as through necrosis.

Granzyme B targets caspase 3 directly or indirectly through the mitochondria, and this initiates the caspase cascade to DNA fragmentation and apoptosis. Caspase activity is required for apoptosis to occur; however, in the absence of caspase activity, granzyme B can still initiate mitochondrial events via the cleavage of Bid. Cleaved Bid translocates to the mitochondria, and then it recruits the proapoptotic Bcl-2 member, Bax, to the mitochondrial membrane, which results in the loss of the $\Delta \Psi_{\mathrm{m}}$ and the release of cytochrome $c$. The granzyme B-mediated $\Delta \Psi_{\mathrm{m}}$ collapse and cytochrome $c$ release are caspase independent, and they result in cell death by necrosis. ${ }^{17}$

In this study, the expression level of granzyme B in the NK/ T-cell lymphoma tissue was correlated with the parameters related to cell death, such as the degree of apoptosis and necrosis, as well as the level of activated caspase 3. In addition, an in vitro study has demonstrated that granzyme B inhibitor suppressed the cleavage of PARP-1 to produce the p85 fragment. PARP-1 is an abundant nuclear protein that is involved in the DNA-base excision-repair system and it is a well-known substrate of caspase $3 .{ }^{18}$ Whereas granzyme B in the necrotic pathway directly cleaves PARP-1 to produce the $64 \mathrm{kDa}$ fragment, ${ }^{19}$ the cleavage of PARP-1 by caspase, as was shown in the present study, yields the signature of the $85 \mathrm{kDa}$ apoptotic fragment. On the other hand, the loss of $\Delta \Psi_{\mathrm{m}}$, which was partly suppressed after administration of granzyme B inhibitor, lends evidence that granzyme B also exerts its function on cell death through the caspase-independent necrotic pathway. Taken together, these observations support the notion that granzyme B participates in the cell death of NK/T-cell lymphoma through both the apoptotic and necrotic pathways. These apoptosis and necrosis were not independent events but two mechanisms that went hand in hand in the cell death of NK/T-cell lymphoma. Prior studies have demonstrated upregulated expression of chemokines in the tissue of NK/T-cell lymphoma as a mechanism for endothelial damage leading to tissue necrosis. ${ }^{3}$ Because necrosis is the common result of an irreversible cell injury from various causes, we suspect that necrosis of NK/T-cell lymphoma is derived from activation of granzyme B as well as vascular damage. In our study, necrotic zone often contained entirely necrotic blood vessels. Some but not all viable blood vessels in necrotic zone showed endothelial damage that may relate to ischemic necrosis of tumor tissue. However, it was difficult 
to determine whether such an endothelial change of blood vessels is the consequence or cause of tissue necrosis, because detection of vascular endothelial damage in non-necrotic zone was not easy because of limited size of nasal biopsy.

Granzyme B is generally confined within the lytic granules; hence, it is not expected to attack the cells in which the granzyme B lies. However, a recent study has reported that granzyme B plays a role in activation-induced T-cell death, which is a specific form of apoptosis that maintains homeostasis of the T-cell population. ${ }^{20}$ Although this form of apoptosis has been thought to require the interaction of death receptors with their ligands, such as the Fas-FasL system, accumulating evidence from a number of recent studies also suggests that blocking of the death receptor pathways can result in activation-induced cell death via the direct cleavage of caspase 3 by granzyme B. ${ }^{21}$

Using the NK/T-cell lymphoma cell line, we demonstrated granzyme B leakage into the cytosol of the HANK-1 cell line. Granzyme B was located within the cytosol as well as in the cytolytic granules. The intrinsic granzyme B inhibitor, PI-9, which is in a separate subcellular compartment from granzyme B, formed an SDS-resistant complex with granzyme B. These findings are very similar to those described in a recent study on the activation-induced cell death of NK cells. Ida $e$ t $a l^{22}$ described that activation of IL-2-primed NK cells, by anti-CD2, induced cell death, and this was accompanied by leakage of granzyme B from the intracellular granules into the cytosol. In our study, the NK/T-cell line was cultured in media supplemented with IL-2, because the HANK-1 cell line is dependent on IL-2 for survival. ${ }^{11}$ The effect of IL-2 on cytotoxic $\mathrm{T}$ cells includes increased lytic activity, induction of lymphokine-activated killer activity, stimulation of IFN- $\gamma$ production, and also cell proliferation. In addition, IL-2 directly regulates granzyme gene expression in CD8 $+\mathrm{T}$ cells independently of its effects on cell survival and proliferation. $^{23}$

Most of the NK/T-cell lymphomas are latently infected by EBV. The LMP-1 gene of EBV can upregulate such Th-1 cytokines as IFN- $\gamma$ and TNF- $\alpha$ via TRAF2/5-NF- $\kappa$ B signals. ${ }^{24}$ Accordingly, in vivo NK/T-cell lymphomas upregulate many cytokine genes, predominantly the Th-1 type, as was demonstrated by a recent DNA microarray analysis by Ohshima et al. $^{25}$ The commonly upregulated cytokine genes include IP-10, IFN- $\gamma$, Mig, and MCP-1, 2, 3, and 4. These cytokines, and especially IP-10 and Mig, are known to enhance NK-cellmediated cell lysis and the release of granule-derived serine esterases. ${ }^{7}$ Under this cytokine-rich environment, the cytotoxic granules in NK/T-cell lymphoma seem to be easily activated, leaked into the cytosol, and induce cell death by either a caspase-dependent (apoptosis) or caspase-independent (necrosis) pathway. This results in the prominent cell death of tumor cells, which is a common histologic change in EBV-associated lymphoproliferative disease.

The cytokine storm in NK/T-cell lymphoma induces hemophagocytic syndrome, multiorgan failure, and a relentless progression of disease. Considering the correlation between the extent of tumor necrosis and worse prognosis of the patients, as shown in the previous study, ${ }^{26}$ the EBV-induced cytokine secretion appears to be an important pathogenetic factor that determines the clinicopathologic features of NK/T-cell lymphoma.

In conclusion, our results suggest that activation of NK/T-cell lymphoma by cytokines leads to the leakage of granzyme B, which initiates the self-induced death of tumor cells through both caspase-dependent and caspaseindependent pathways.

\section{ACKNOWLEDGEMENT}

This study was supported by SBRI grants C-A3-201-1 and 2, and C-A3-202-3.

1. Jaffe ES, Harris NL, Stein $\mathrm{H}$, et al. World Health Organization Classification of Tumours, Pathology \& Genetics Tumours of the Haematopoietic and Lymphoid Tissues. International Agency for Research on Cancer (IARC) Press: Lyon, 2001, pp 214-215.

2. Lipford Jr EH, Margolick JB, Longo DL, et al. Angiocentric immunoproliferative lesions: a clinicopathologic spectrum of postthymic T-cell proliferations. Blood 1988;72:1674-1681.

3. Teruya-Feldstein J, Jaffe ES, Burd PR, et al. The role of Mig, the monokine induced by interferon-gamma, and IP-10, the interferongamma-inducible protein-10, in tissue necrosis and vascular damage associated with Epstein-Barr virus-positive lymphoproliferative disease. Blood 1997;90:4099-4105.

4. $\mathrm{Ng} \mathrm{CS}$, Lo ST, Chan JK. Peripheral $\mathrm{T}$ and putative natural killer cell lymphomas commonly coexpress CD95 and CD95 ligand. Hum Pathol 1999;30:48-53.

5. Takakuwa T, Dong Z, Nakatsuka S, et al. Frequent mutations of Fas gene in nasal NK/T cell lymphoma. Oncogene 2002;21:4702-4705.

6. Jeon YK, Kim H, Park SO, et al. Resistance to Fas-mediated apoptosis is restored by cycloheximide through the downregulation of cellular FLIPL in NK/T-cell lymphoma. Lab Invest 2005;85:874-884.

7. Taub DD, Sayers TJ, Carter CR, et al. Alpha and beta chemokines induce NK cell migration and enhance NK-mediated cytolysis. J Immunol 1995;155:3877-3888.

8. Lord SJ, Rajotte RV, Korbutt GS, et al. Granzyme B: a natural born killer. Immunol Rev 2003;193:31-38.

9. Cho MS, Lee MK, Yang Wl, et al. Granzyme B immunoreactivity in T/natural killer cell lymphomas. Yonsei Med J 1997;38:285-293.

10. Sun J, Bird CH, Sutton V, et al. A cytosolic granzyme B inhibitor related to the viral apoptotic regulator cytokine response modifier $A$ is present in cytotoxic lymphocytes. J Biol Chem 1996;271:27802-27809.

11. Kagami Y, Nakamura S, Suzuki R, et al. Establishment of an IL-2dependent cell line derived from 'nasal-type' NK/T-cell lymphoma of CD2+, sCD3-, CD3epsilon+, CD56+ phenotype and associated with the Epstein-Barr virus. Br J Haematol 1998;103:669-677.

12. Robertson MJ, Cochran KJ, Cameron C, et al. Characterization of a cell line, NKL, derived from an aggressive human natural killer cell leukemia. Exp Hematol 1996;24:406-415.

13. Morales-Gonzalez JA, Gutierrez-Salinas J, Pina E. Release of mitochondrial rather than cytosolic enzymes during liver regeneration in ethanol-intoxicated rats. Arch Med Res 2004;35:263-270.

14. Kerr JFR, Wyllie AH, Currie AR. Apoptosis: a basic biological phenomenon with wide-ranging implications in tissue kinetics. $\mathrm{Br} \mathrm{J}$ Cancer 1972;26:239-257.

15. Jäättelä $M$, Leist $M$. From caspases to alternative cell-death mechanisms. In: Yin XM, Dong Z (eds). Essentials of apoptosis. A Guide for Basic and Clinical Research. Humana Press Inc.: New Jersey, 2003, pp 101-122.

16. Yeldandi A, Kaufman DG, Reddy JK. Cell injury and cellular adaptations. In: Damjanov I, Linder J (eds). Anderson's pathology, 10th edn. Mosby: St Louis, Missouri, 1996, pp 357-386.

17. Lord SJ, Rajotte RV, Korbutt GS, et al. Granzyme B: a natural born killer. Immunol Rev 2003;193:31-38. 
18. Kam CM, Hudig D, Powers JC. Granzymes (lymphocyte serine proteases): characterization with natural and synthetic substrates and inhibitors. Biochim Biophys Acta 2000;1477:307-323.

19. Gobeil $S$, Boucher CC, Nadeau D, et al. Characterization of the necrotic cleavage of poly(ADP-ribose) polymerase (PARP-1): implication of lysosomal proteases. Cell Death Differ 2001;8:588-594.

20. Gorak-Stolinska P, Truman JP, Kemeny DM, et al. Activation-induced cell death of human T-cell subsets is mediated by Fas and granzyme $B$ but is independent of TNF-alpha. J Leukoc Biol 2001;70:756-766.

21. Atkinson EA, Barry M, Darmon AJ, et al. Cytotoxic T lymphocyteassisted suicide. Caspase 3 activation is primarily the result of the direct action of granzyme B. J Biol Chem 1998;273:21261-21266.

22. Ida H, Nakashima T, Kedersha NL, Yamasaki S, et al. Granzyme B leakage-induced cell death: a new type of activation-induced natural killer cell death. Euro J Immunol 2003;33:3284-3292.
23. Janas $\mathrm{ML}$, Groves $\mathrm{P}$, Kienzle $\mathrm{N}$, et al. IL-2 regulates perforin and granzyme gene expression in CD8+ T cells independently of its effects on survival and proliferation. J Immunol 2005;175: 8003-8010.

24. Chuang HC, Lay JD, Hsieh WC, et al. Epstein-Barr virus LMP1 inhibits the expression of SAP gene and upregulates Th1 cytokines in the pathogenesis of hemophagocytic syndrome. Blood 2005;106: 3090-3096.

25. Ohshima K, Karube K, Hamasaki M, et al. Differential chemokine, chemokine receptor and cytokine expression in Epstein-Barr virusassociated lymphoproliferative diseases. Leuk Lymphoma 2003;44:1367-1378.

26. Ko YH, Cho EY, Kim GE, et al. NK and NK-like T-cell lymphoma in extranasal sites: a comparative clinicopathological study according to site and EBV status. Histopathology 2004;44:480-489. 